\title{
Diagnóstico situacional e intervenções de terapia ocupacional em unidade de terapia intensiva onco-hematológica pediátrica
}

\section{Situational diagnosis and occupational therapy contributions in a pediatric onco- hematologic intensive care unit \\ Diagnóstico situacional e intervención de terapia ocupacional en la unidade de cuidados intensivos pediátricos onco-hematológico}

\section{Recebido: 05/06/2017 \\ Aprovado: 15/11/2017 \\ Publicado: 30/03/2018}

\author{
Mariana de Paiva Franco ${ }^{1}$ \\ Aide Mitie Kudo ${ }^{2}$
}

O objetivo deste estudo foi traçar o diagnóstico situacional de uma Unidade de Terapia Intensiva Oncohematológica Pediátrica (UTIOP) e verificar as possíveis intervenções de Terapia Ocupacional (TO). Este é um relato de experiência retrospectivo e descritivo, realizado em 2014 considerando o período de quatro meses após a inserção da TO na UTIOP de um Hospital terciário da cidade de São Paulo. A partir da compreensão do perfil dos pacientes e da dinâmica da Unidade, foi possível a elaboração de um instrumento de avaliação. Para o período a T0 atendeu 25 de 29 crianças internadas, com predominância de idade de até 7 anos e diagnóstico de leucemia, com a condição de internação de consciente ou sedado e tempo de internação de até 7 dias. A TO realizou 97 intervenções: avaliação, orientações aos cuidadores, posicionamento no leito, Tecnologia Assistiva. A partir da experiência clínica pode se afirmar que o TO possui competência para intervir junto aos pacientes e seus familiares no espaço da Unidade de Terapia Intensiva Pediátrica e Oncológica, contribuindo significativamente com a equipe multiprofissional e na qualidade de vida dos pacientes/cuidadores assistidos.

Descritores: Terapia ocupacional; Unidades de terapia intensiva pediátrica; Serviço Hospitalar de Oncologia.

The objective of this study was to trace the situational diagnosis of a Pediatric Onco-hematological Intensive Care Unit (POICU) and verify the possible interventions of Occupational Therapy (OT). This is a retrospective and descriptive experience report carried out in 2014, considering the period of four months after insertion of OT in the POICU of a tertiary hospital in the city of São Paulo. Based on the understanding of the patients' profile and the dynamics of the Unit, it was possible to create an assessment instrument. For the period studied, 25 out of 29 hospitalized children aged over 7 years and diagnosed with leukemia were admitted to the hospital in conscious or sedated state at the moment of hospitalization, and the length of hospitalization was up to 7 days. Occupational Therapy performed 97 interventions: assessment, guidance to caregivers, bed positioning, Assistive Technology. The clinical experience made it possible to affirm that OT has competence to intervene in the case of patients and families in the space of Pediatric Onco-hematological Intensive Care Unit, significantly contributing to the multiprofessional team and to the quality of life of the patients/careers assisted.

Descriptors: Occupational therapy; Intensive care units pediatric; Oncology service hospital.

El objetivo de este estudio fue trazar el diagnostico situacional de una Unidad de Terapia Intensiva Oncohematológica Pediátrica (UTIOP) y verificar las posibles intervenciones de Terapia Ocupacional (TO). Este es un relato de experiencia retrospectivo y descriptivo, realizado en 2014 considerando el período de cuatro meses después de la inserción de la TO en la UTIOP de un Hospital terciario de la ciudad de São Paulo. A partir de la comprensión del perfil de los pacientes y de la dinámica de la Unidad, fue posible la elaboración de un instrumento de evaluación. Para el periodo la TO atendió 25 de 29 niños internados, con predominio de edad de hasta 7 años y diagnóstico de leucemia, con la condición de internación de consiente o sedado y tiempo de internación de hasta 7 días. La To realizó 97 intervenciones: evaluación, orientaciones a los cuidados, posición en el lecho, Tecnología Asistida. A partir de la experiencia clínica se puede afirmar que el TO posee competencia para intervenir junto a los pacientes y de sus familiares en el espacio de la Unidad de Terapia Intensiva Pediátrica y Oncológica, contribuyendo significativamente con el equipo multiprofessional y en la calidad de vida de los pacientes/cuidadores asistidos.

Descriptores: Terapia ocupacional; Unidades de cuidado intensivo pediátrico; Servicio de oncología en hospital.

1Terapeuta Ocupacional. Especialista em Saúde Mental. Especialista en Intervenção Neuro-pediátrica. ORCID: 0000-0001-7933-1256 E-mail: mariana.franco@hc.fm.usp.br

2 Terapeuta Ocupacional. Especialista em Administração Hospitalar. Supervisora do Serviço Hospitalar y Coordenadora do Serviço de Terapia Ocupacional do Instituto da Criança do Hospital de Clínicas da Faculdade de Medicina da Universidade de São Paulo. ORCID: 0000-0003-2233-5701 E-mail: aide.kudo@hc.fm.usp.br 


\section{INTRODUÇÃO}

$\mathbf{0}$ câncer em crianças e adolescentes entre 0 e 19 anos, é um conjunto de doenças que apresenta características próprias, principalmente com relação ao comportamento clínico e à histopatologia.

É considerada rara, correspondendo entre $1 \%$ e $3 \%$ de todos os tumores malignos. No Brasil, mortes de crianças e jovens causadas pelo câncer representam 7\%, sendo considerada a maior causa de morte ligada a doenças. Em 2013, ocorreram 2.835 mortes ligadas ao câncer de crianças e jovens de $1 \mathrm{a}$ 19 anos. Os diagnósticos de neoplasias mais comuns na infância são as leucemias, tumores do sistema nervoso central, linfomas, neuroblastoma, tumor de Wilms, retinoblastoma, tumor germinativo, ostessarcoma e sarcomas ${ }^{1}$.

Nos últimos 40 anos, o progresso no tratamento do câncer na infância e adolescência foi significativo, ocorrendo sucesso no tratamento em $70 \%$ dos casos, quando diagnosticados precocemente e tratados em centros especializados ${ }^{2}$.

0 crescente avanço do conhecimento científico, da tecnologia no diagnóstico e dos recursos terapêuticos, tem contribuído na eficácia do tratamento de crianças portadoras de patologias onco-hematológicas, contudo a própria evolução da doença e complicações clínicas com risco de morte durante o tratamento ainda é uma realidade. Nessa fase, os recursos da unidade de terapia intensiva são fundamentais para superar essa etapa crítica do tratamento ${ }^{3}$.

A Unidade de Terapia Intensiva caracteriza-se por uma unidade com estrutura capaz de prestar assistência especializada a pacientes grave, em estado crítico que necessitam de controles rigorosos $\mathrm{e}$ intensivos dos parâmetros vitais e que apresentam potencial risco de morte ${ }^{4}$. Estes fatos requerem atenção multiprofissional especializada de forma contínua, materiais específicos e tecnologias necessárias ao diagnóstico, monitorização e terapia.

Quanto a Unidade de Terapia Intensiva Pediátrica, ela não difere da Unidade de Adulto, e é definida, segundo a Resolução no 7 , de 24 de fevereiro de 2010, do Ministério da
Saúde, como área crítica destinada à internação de pacientes graves com idade de 29 dias a 14 ou 18 anos, sendo este limite variável de acordo com cada instituição ${ }^{5}$.

Devido a essas condições, o paciente em terapia intensiva, normalmente é um paciente: clinicamente grave/crítico; hemodinamicamente instável; com nível de consciência alterado (podendo estar vigil, em coma ou coma induzido); em monitorização contínua dos sinais vitais; com necessidade de ventilação mecânica (para pacientes com insuficiência respiratória); com acessos venosos periféricos ou central para infusão de medicações, drenos e sondas; manipulado constantemente, pelos profissionais, devido aos procedimentos clínicos invasivos, controles e medicações; e, restrito ao leito ${ }^{3}$.

Quanto ao ambiente físico, a UTI é caracterizada por ser uma unidade fechada, restrita e limitada para acesso das pessoas; com os leitos podendo ser separados por divisórias, dispostos de maneira que possibilitem observação constante pelos profissionais; utilização de diversos equipamentos de monitorização e de suporte básico de vida, como ventiladores mecânicos (VM), monitores de sinais vitais, equipamentos para infusão contínua e controle de fluidos; iluminação, normalmente, artificial, contínua e coletiva; com ruídos intermitentes devido aos equipamentos com alertas sonoros ${ }^{3}$.

Ainda em relação à Resolução nº 7, de 24 de fevereiro de 2010, do Ministério da Saúde, o $18^{\circ}$ artigo, recomenda que uma Unidade de Terapia Intensiva Adulta e Pediátrica, deve garantir a assistência de Terapia Ocupacional por meios próprios ou terceirizados 5 .

Diante disso, o terapeuta ocupacional inserido na equipe multiprofissional da Unidade de Terapia Intensiva pode contribuir com a atenção integral ao paciente e seu familiar.

0 olhar do terapeuta ocupacional considerando o indivíduo em seus aspectos pessoal, social e afetivo pode minimizar os sofrimentos causados pelo processo de adoecimento e internação utilizando os recursos terapêuticos ocupacionais 
adequados ao ambiente de terapia intensiva e as condições clínicas do paciente ${ }^{6}$.

A Terapia Ocupacional atua na prevenção e promoção de ações para a manutenção dos desempenhos ocupacionais que possibilitam a melhora da saúde, qualidade de vida e autonomia do paciente, responsável por analisar e promover a vida ocupacional do paciente em seus diferentes aspectos ${ }^{7}$.

Em contexto hospitalar pediátrico, a atuação da Terapia Ocupacional está voltada para a promoção da saúde, buscando alternativas para potencializar a qualidade de vida do paciente e ressignificação do cotidiano que foi interrompido, em função do adoecimento e da internação ${ }^{8}$.

A atuação profissional neste contexto deve abranger aspectos físicos, orgânicos e funcionais, assim como os do âmbito psicossocial inerentes ao curso da doença, tratamento e hospitalização, como dor e ruptura do cotidiano, risco eminente de morte, assim como as expectativas dos pacientes e familiares a respeito do tratamento, cuidados e impactos em seu cotidiano e rotina intra e extra-hospitalar7.

Neste sentido, a atuação é ampla, abordando aspectos físicos da funcionalidade; prevenção de ulceras por pressão em função da imobilidade no leito; auxilio na comunicação e interação do paciente; e aspectos emocionais decorrentes da clínica do paciente e do ambiente estressor da unidade de terapia intensiva.

Assim, 0 objetivo deste estudo foi traçar o diagnóstico situacional de uma Unidade de Terapia Intensiva Oncohematológica Pediátrica (UTIOP) e verificar as possíveis intervenções de TO.

\section{MÉTODO}

0 presente estudo caracteriza-se como um relato de experiência que busca apresentar a análise retrospectiva sobre a atuação da Terapia Ocupacional, realizada no período de abril/2014 a agosto/2014, durante o início da assistência terapêutica ocupacional em uma
Unidade de Terapia Intensiva, inserida na Unidade de Onco-hematologia Pediátrica de um Hospital Terciário, especializado, vinculado a uma Instituição de Ensino Superior da cidade de São Paulo.

0 processo de inserção da Terapia Ocupacional iniciou na primeira quinzena de abril de 2014, concomitantemente com a inauguração da referida Unidade. Em agosto de 2014, julgou-se necessário realizar uma análise retrospectiva, traçando um diagnóstico situacional da Unidade Intensiva e buscando compreender o perfil dos pacientes, dinâmica da unidade e as possíveis intervenções terapêuticas ocupacionais neste contexto.

Trata-se de um estudo descritivo, realizado por meio de observações clínicas, registros de internações, dados pessoais e clínicos contidos em prontuário e registro de intervenções realizadas pela terapeuta ocupacional de referência da Unidade e, de discussão clínica com equipe multiprofissional. Os dados foram analisados e apresentados em forma de gráficos.

\section{RESULTADOS}

As atividades da UTIOP tiveram início na segunda quinzena de abril de 2014, com capacidade operacional para sete leitos, mas somente com três leitos ativos no período da análise.

0 público alvo foram pacientes de 0 a 18 anos com diagnóstico de doenças Oncohematológicas ou que tinham sido submetidas a Transplante de Células Tronco Hematopoéticas (TCTH). Neste período $\left(2^{\mathrm{a}}\right.$ quinzena de abril a $1^{\text {a }}$ quinzena de agosto de 2014), a unidade prestou assistência a 29 pacientes.

A inserção da Terapia Ocupacional neste contexto, ocorreu concomitantemente com a inauguração da Unidade e neste período, foram atendidos pelo Serviço de Terapia Ocupacional, 25 pacientes, com idade entre 5 meses e 22 anos, com predominância de 0 a 8 anos (Gráfico 1). 


\section{Faixa etária}

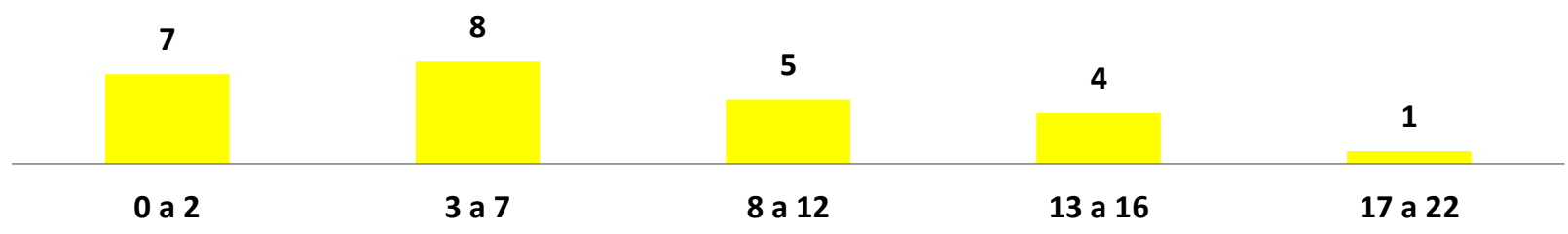

Gráfico .1 Faixa etária de internados UTI pediátrica entre abril a agosto de 2014. HC-FMUSP, 2014.

Quanto ao diagnóstico de base, (4\%), anemia hemolítica auto imune (AHAI) observou-se as seguintes patologias: (4\%), adrenoleucodistrofia (4\%), síndrome leucemias (LMA e LLA) (40\%), tumores do da imunodeficiência combinada (SCID) (4\%), SNC (28\%) - dentre estes, o meduloblastoma osteopetrose (4\%) e histiocitose (4\%), teve a maior prevalência (16\%) - , conforme o Gráfico 2. neuroblastoma $(8 \%)$, seguidos por linfoma

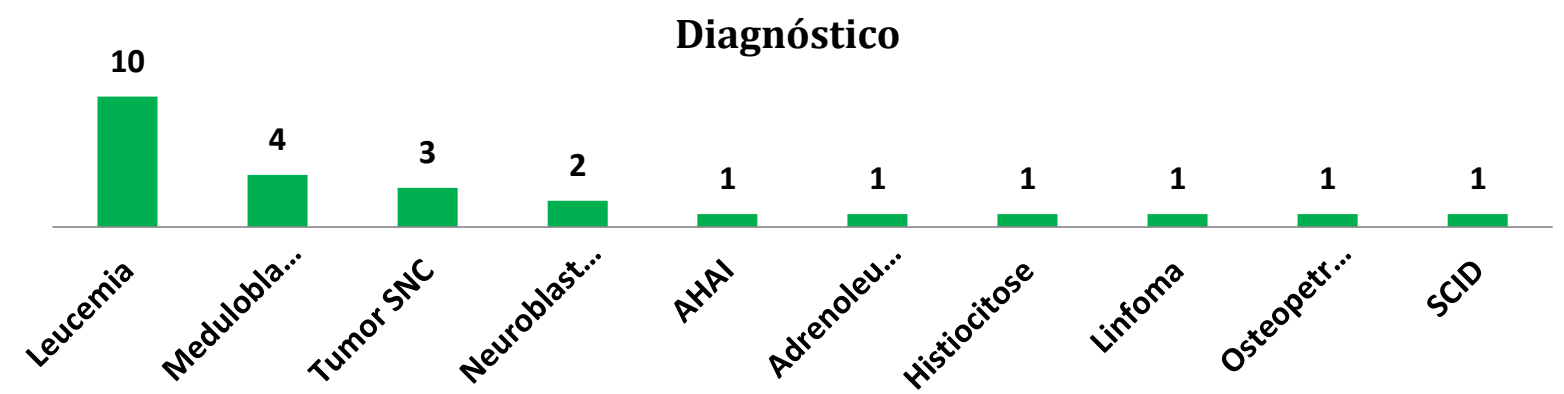

Gráfico 2. Diagnóstico de internados UTI pediátrica entre abril a agosto de 2014. HC-FMUSP, 2014.

Os motivos para transferência para UTIOP foram: sepses (28\%), risco de lise tumoral (16\%), rebaixamento do nível de consciência (12\%), complicações após TCTH (12\%), abdome agudo (8\%), seguidos por risco de hemorragias (4,8\%), acidente vascular encefálico (AVE) $(4,8 \%)$, hipertensão refratária $(4,8 \%)$, mal convulsivo $(4,8 \%)$ e complicações respiratória $(4,8 \%)$, como no Gráfico 3.

\section{Motivos da internação}

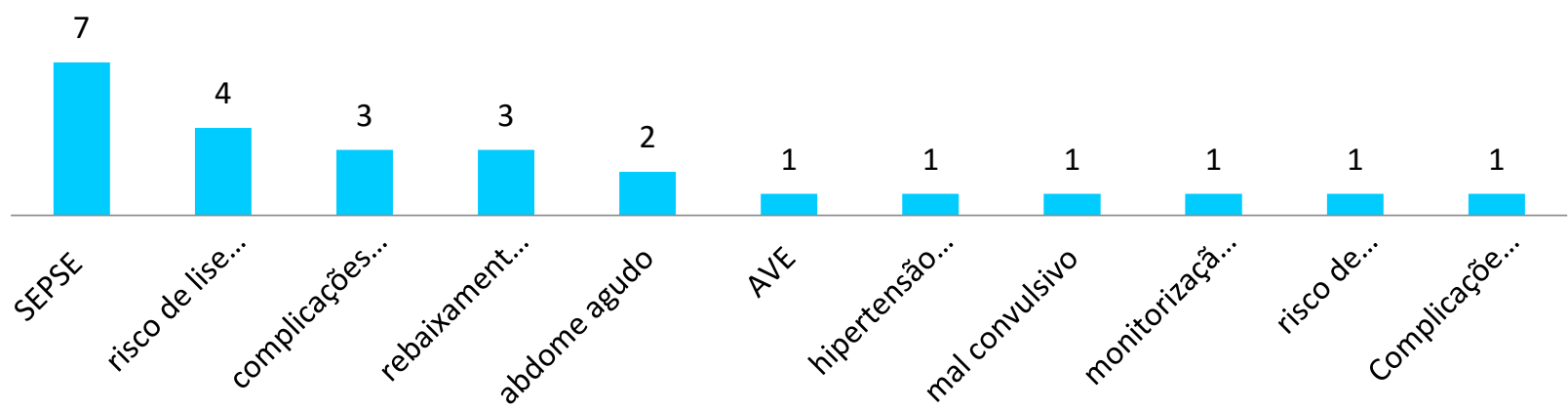

Gráfico 3. Motivos de internação UTI pediátrica entre abril a agosto de 2014. HC-FMUSP, 2014.

Nas condições apresentadas pelos pacientes durante as intervenções no quesito comunicação/interação, classificou-se em quatro categorias: contactuante; sedado, rebaixamento do nível de consciência; e, sedado/contactuante. $\mathrm{Na}$ categoria 1 contactuante, foram incluídos os pacientes com possibilidade de comunicação verbal ou não verbal. Na categoria 2 - rebaixamento do nível de consciência, aqueles que não estabelecem nenhum tipo de comunicação devido ao nível de consciência rebaixado. $\mathrm{Na}$ categoria 3 - sedado, aqueles que não estabelecem contato devido à necessidade do 
uso de medicações para sedação devido à condição clínica e/ou necessidade de intubação orotraqueal (IOT). Já na categoria 4 - sedado/ contactuante, aqueles que a terapeuta ocupacional realizou intervenções em dois momentos distintos, durante o período de sedação e após extubação, conforme o Gráfico 4.

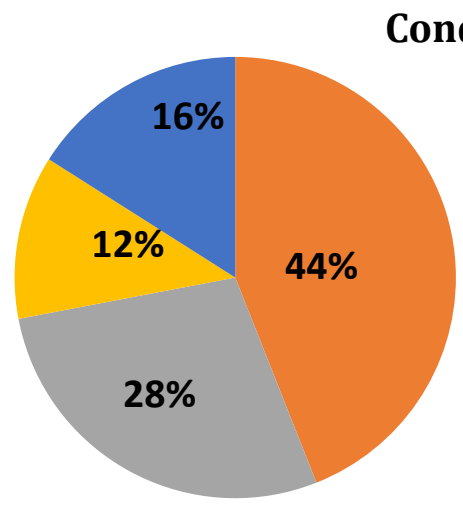

\author{
contactuante \\ sedado \\ rebaixamento nivel de \\ consciencia \\ n sedado / contactuante
}

Gráfico 4. Condições de internação UTI pediátrica entre abril a agosto de 2014. HC-FMUSP, 2014.

Quanto ao tempo de permanência na UTIOP dos pacientes atendidos pela Terapia Ocupacional houve uma variável entre 1 e 98 dias. Tendo uma maior prevalência entre 1 a 7 dias, representado por $52 \%$ dos casos, seguidos por $24 \%$ no período entre 8 a 14 dias, $16 \%$ entre 15 a 21 dias e $4 \%$ entre 22 a 28 dias e o mesmo percentual para até 98 dias de permanência na unidade, como no Gráfico 5 .

\section{Tempo de permanência (dias)}

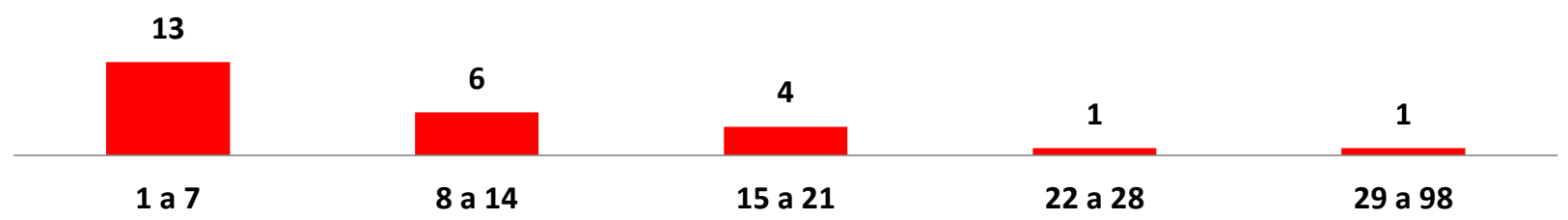

Gráfico 5. Dias de internação na UTI pediátrica entre abril a agosto de 2014. HC-FMUSP, 2014.

Nas formas de alta ou saída houveram três tipos: óbito, para a residência e transferências. As transferências se deram para outras unidades vinculadas ao referido hospital e retorno ao serviço de origem, conforme Gráfico 6.

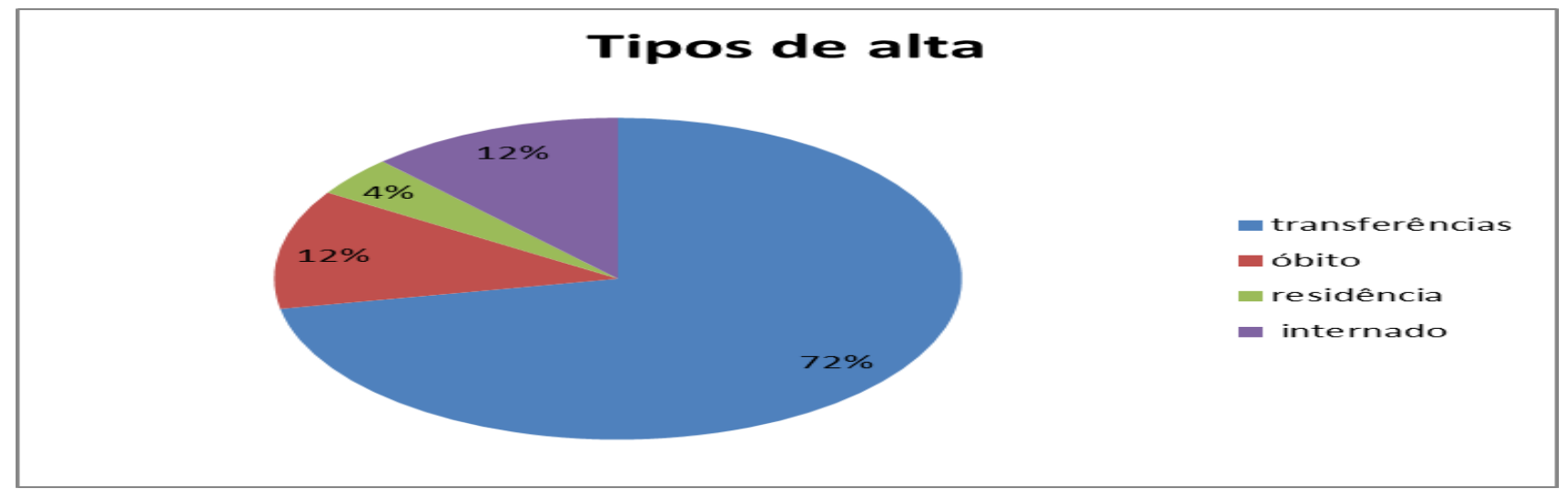

Gráfico 6. Tipos alta-saída da UTI pediátrica entre abril a agosto de 2014. HC-FMUSP, 2014. 
No período analisado, foram realizadas 97 intervenções de Terapia Ocupacional e se constituíram por: avaliação, orientações aos cuidadores, posicionamento no leito, Tecnologia Assistiva - avaliação e confecção de órtese de MMSS e adaptação para o brincar; realização de atividade de interesse e acolhimento de pais ou acompanhantes.

\section{DISCUSSÃO}

Os achados neste estudo em relação ao, que aponta a leucemia (40\%) e os tumores do sistema nervoso central (SNC) como os principais diagnósticos dos pacientes atendidos nesta Unidade, vão de encontro ao que é descrito pelo INCA, 2017, onde referem que as leucemias representam o maior percentual (26\%), seguida dos linfomas (14\%) e tumores do sistema nervoso central na população pediátrica; assim como, a estimativa de ocorrência de 12.600 novos casos de câncer na faixa etária de zero a 19 anos em $2017^{9}$.

Em relação às condições de interação apresentados pelos pacientes, observa-se que, a maioria dos pacientes se enquadrou na categoria 1 - contactuantes (44\%), o que possibilitou uma grande variação de estratégias para o atendimento terapêutico ocupacional, porém, vale ressaltar que, devido a ausência de unidade de internação semi intensiva neste serviço, pacientes com demandas para este tipo de cuidado, internaram na UTIOP, podendo, ocorrer alterações neste perfil após a implantação da unidade semi intensiva.

Um formulário de avaliação foi elaborado com objetivo de identificar demandas para acompanhamento do paciente e/ ou familiares, considerando às necessidades apresentadas pelo paciente e/ou cuidador, as condições clínicas e/ ou das condições de comunicação e interação dos pacientes.

Alguns aspectos fundamentais para a avaliação no contexto da terapia ocupacional ${ }^{3}$ : - histórico ocupacional;

- rede familiar/social de suporte;

- impacto da internação (paciente/cuidador);

- alteração no desempenho ocupacional;

- histórico do adoecimento;
- condições clínicas;

- nível de consciência;

- alterações percepto-cognitivas;

- alterações neuromúsculoesqueléticas;

- desenvolvimento neuropsicomotor.

A avaliação engloba aspectos físicos e emocionais do paciente e deve nortear a linha para estabelecer o programa terapêutico ocupacional. Na UTI, a conduta de Terapia Ocupacional deve ser centrada no estado clínico, funcional, emocional e cognitivo do paciente. A consulta prévia ao prontuário anterior à intervenção é fundamental para observar o histórico clínico prévio, como cirurgias e hospitalizações ${ }^{10}$.

A partir da apropriação da rotina e do entendimento dos equipamentos específicos inseridos no contexto da UTI, o terapeuta ocupacional, sente-se mais seguro, com possibilidades de ampliação de sua prática, identificando estratégias de intervenções, apesar da instabilidade clínica e gravidade dos pacientes atendidos ${ }^{11}$.

Os principais objetivos de intervenções da terapia ocupacional em uma UTIP são ${ }^{3}$ :

\section{Pacientes}

- ressignificação do cotidiano;

- minimização dos impactos da internação na uti;

- prevenção de eventuais deformidades, edemas, pontos de pressão e dor (pacientes sedados ou vigil - restritos ao leito);

- manutenção do desempenho ocupacional;

- manutenção da capacidade funcional;

- vigilância do desenvolvimento neuropsicomotor (bebês);

- assistência aos pacientes e cuidadores em processo de terminalidade.

\section{Ambiente}

- orientação temporo espacial;

- privacidade;

- individualidade;

- redução de estímulos adversos (ruídos, iluminação).

\section{Cuidadores}

- Orientação, acolhimento e empoderamento.

A intervenção precoce e intensiva de Terapia Ocupacional é efetiva na prevenção do delirium em adultos hospitalizados, reduz o seu tempo de internação e aumenta o nível 
de independência funcional motora por ocasião de sua alta12.

0 ambiente de uma UTI é marcado por ser um local adverso e pouco acolhedor, pelos ruídos dos equipamentos, necessidade de procedimentos e práticas invasivas, nível elevado de estresse ${ }^{11}$.

Porém ressalta-se a respeito das possibilidades de intervenção voltada para este âmbito, sendo elas, desde a conscientização da equipe em relação à redução de ruídos, até a realização de atividades visando à orientação temporo espacial destes pacientes após períodos prolongados neste contexto.

Por se tratar de uma unidade pediátrica, o Estatuto da Criança de do Adolescente (ECA), preconiza a presença de um acompanhante de referência da criança durante todo o período de internação ${ }^{13}$. Diante disso, dentro de uma UTI pediátrica, se encontra acompanhantes adultos em todos os períodos de um dia, sendo necessário que haja um olhar atento da equipe multiprofissional para esses acompanhantes.

A intervenção de TO também deve abranger o atendimento aos pais, pois a família de uma criança internada em Unidade de Terapia Intensiva também vivencia o sofrimento da hospitalização e incerteza quanto ao quadro clínico do paciente ${ }^{14}$.

Julga-se necessário também, a inclusão dos cuidadores, quando possível, nos cuidados menos complexos com a criança, visando à manutenção do vínculo e a manutenção dos papéis ocupacionais, descrito pela AOTA, como uma das atividades instrumentais da vida diária (AIVDs) ${ }^{15}$.

Como estratégia de intervenção aos cuidadores, a implantação de grupos de acompanhantes é um espaço que permite aos pais compartilhar sentimentos e experiências que envolvem todo o processo de internação em uma UTI ${ }^{14}$.

No ambiente de UTI, os acometimentos que os pacientes internados apresentam (gravidade do quadro, necessidade de suporte ventilatório, sedados, traqueostomizados, com ulcera por pressão, nível de consciência alterado) podem dificultar as intervenções da equipe multiprofissional, dentre eles a da
Terapia Ocupacional, podendo gerar angustias e impotência ${ }^{11}$.

\section{CONCLUSÃO}

0 terapeuta ocupacional, embora seja considerado como membro da equipe pelo Ministério da Saúde, observa-se baixa inserção destes profissionais neste campo e uma produção científica específica restrita.

A partir de experiência clínica pode se afirmar que o terapeuta ocupacional possui competência para intervir junto aos pacientes e seus familiares no espaço da Unidade de Terapia Intensiva Pediátrica e Oncológica, contribuindo significativamente com a equipe multiprofissional e na qualidade de vida dos pacientes/ cuidadores assistidos.

A atuação do terapeuta ocupacional contribui na garantia da atenção integral ao paciente, proporcionando-lhe qualidade de vida durante o período da internação, englobando os aspectos funcionais e lúdicos, favorecendo o seu desempenho ocupacional.

Em alguns casos a intervenção é focada no acolhimento aos acompanhantes devido às condições clínicas graves dos pacientes, sendo realizado o acolhimento e o empoderamento dos cuidados por meio de orientações durante esse período e pós-alta.

0 presente estudo foi relevante para a identificação do perfil de pacientes atendidos em uma Unidade de Terapia Intensiva OncoHematológica Pediátrica, compreensão deste contexto, inserção do profissional em equipe multiprofissional e contribuições para este campo de atuação profissional, ainda em ampliação.

\section{REFERÊNCIAS}

1. Instituto Nacional de Câncer "José Alencar Gomes da Silva", Coordenação de Prevenção e Vigilância. Estimativa 2016: incidência de câncer no Brasil. Rio de Janeiro: INCA; 2015. 122p.

2. Instituto Nacional de Câncer "José Alencar Gomes da Silva”, Coordenação de Prevenção e Vigilância de Câncer. Câncer da criança e adolescente no Brasil: dados dos registros de base populacional e de mortalidade. Rio de Janeiro: INCA; 2008. 220 p.

3. Franco MP, Kudo AM. Intervenção de terapia ocupacional em unidade de terapia intensiva pediátrica. In: De Carlo MMRP, Kudo AM, organizadores. Terapia ocupacional em contextos 
hospitalares e cuidados paliativos. São Paulo: Payá; 2017. (no prelo).

4. Fernandes HS, Silva E, Capone Neto A, Pimenta LA, Knobel E. Gestão em terapia intensiva: conceitos e inovações. Rev Bras Clin Med. 2011; 9(2):129-379.

5. Agência Nacional de Vigilância Sanitária. Resolução RDC no 7, de 24 de fevereiro de 2010. Dispõe sobre os requisitos mínimos para funcionamento de Unidades de Terapia Intensiva e dá outras providências. D.O.U., Brasília, 25 fev 2010 Disponível

em: http://bvsms.saude.gov.br/bvs/saudelegis/anvisa/ 2010/res0007_24_02_2010.html.

6. Albuquerque VB. Atuação do terapeuta ocupacional em unidade de terapia intensiva pediátrica junto ao paciente e à família: relato de caso. Rev Saúde Criança Adolesc. 2011; 3(1):63-5.

7. Bigatao MR, Mastroprieto AP, Carlo MMRP. Terapia ocupacional em oncologia: a experiência do Hospital das Clínicas da Faculdade de Medicina de Ribeirão Preto da Universidade de São Paulo. In: Othero MB., organizador. Terapia ocupacional: práticas em oncologia. São Paulo: Roca; 2009. p. 302322.

8. Kudo AM, Souza FDA, Franco MP, Bullara P, Barros PBM. Cuidados paliativos em pediatria e o impacto da hospitalização no quotidiano da criança e do adolescente com doença crónica. In: Costa A, Othero MB. Reabilitação em cuidados paliativos. Loures, Portugal: Lusodidacta; 2014. p.289-301.

9. Instituto Nacional de Câncer "José Alencar Gomes da Silva". Câncer infanto-juvenil é tema do Dia Mundial do Câncer 2017 [Internet). Rio de Janeiro: INCA; 2017 [citado em 02 fev 2017]. Disponível em: http://www2.inca.gov.br/wps/wcm/connect/agen cianoticias/site/home/noticias/2017/cancer_infant o-juvenil e tema do dia mundial docancer_2017.
10. Afflek AT, Lieberman S, Polon J, Rohrkemper K. Providing occupational therapy in an intensive care unit. Am J Occup Ther. 1986; 40:323-32.

11. Bombarda TB, Lanza AL, Santos CAV, Joaquim RHT. Terapia ocupacional na unidade de terapia intensiva (UTI) adulto e as percepções da equipe. Cad Ter Ocup UFSCar. 2016; 24(4):827-35.

12. Alvarez E, Garrido M, González F, Guzmán E, Donoso T, Gallegos $S$, et al. Terapia ocupacional precoz e intensiva en la prevención del delirium en adultos mayores ingresados a unidades de paciente crítico: ensayo clínico randomizado: resultados preliminares. Rev Chilena Ter Ocup. 2012; 12(1):4558.

13. Presidência da República (Brasil). Lei no 8.069 de 13 de julho de 1990. Dispõe sobre o Estatuto da Criança e do Adolescente e dá outras providências [Internet]. D.O.U., Brasília, DF, 16 jul 1990 [citado em 23 jan 2014].

Disponível

em: http://www.planalto.gov.br/ccivil_03/LEIS/L8069. htm.

14. Silva FR, Ballarin MLGS, Oliveira JC. Grupo de acompanhantes de crianças internadas em unidade de terapia intensiva. Cad Ter Ocup UFSCar. 2015; 23(4):871-7.

15. Associação Americana de Terapia Ocupacional. Estrutura da prática da terapia ocupacional: domínio e processo. Cavalcanti A, Dutra FCMS, Elui VMC, tradutores. Rev Ter Ocup. 2015; 26(ed esp.):1-49.

CONTRIBUIÇÕES
Mariana de Paiva Franco foi responsável pela
redação, organização dos dados e revisão final.
Aide Mitie Kudo contribuiu na concepção,
escrita e revisão final.

\footnotetext{
Como citar este artigo (Vancouver)

Franco MP, Kudo AM. Diagnóstico situacional e intervenções de terapia ocupacional em unidade de terapia intensiva onco-hematológica pediátrica. REFACS [Internet]. 2018 [citado em inserir dia, mês e ano de acesso];6(1):109-116. Disponível em: inserir link de acesso. DOI: inserir link do DOI.

\section{Como citar este artigo (ABNT)}

FRANCO, M. P.; KUDO, A. M. Diagnóstico situacional e intervenções de terapia ocupacional em unidade de terapia intensiva onco-hematológica pediátrica. REFACS, Uberaba, v. 6, n. 1, p. 109-116, 2018. Disponível em: <inserir link de acesso >. Acesso em: inserir dia, mês e ano de acesso. DOI: inserir link do DOI.

\section{Como citar este artigo (APA)}

Franco, M. P. \& Kudo, A. M.(2018). Diagnóstico situacional e intervenções de terapia ocupacional em unidade de terapia intensiva onco-hematológica pediátrica. REFACS, 6(1), 109-116. Recuperado em: inserir dia, mês e ano de acesso de inserir link de acesso. DOI: inserir link do DOI.
} 